\title{
Microfabrication and Test of a Three-Dimensional Polymer Hydro-focusing Unit for Flow Cytometry Applications
}

\author{
Ren Yang ${ }^{1}$, Daniel L. Feeback ${ }^{2}$, and Wanjun Wang ${ }^{1}$ \\ Department of Mechanical Engineering \\ Louisiana State University \\ Email: wang@1su.edu \\ Phone: 225-578-5807 \\ Fax: 225-578-5924 \\ ${ }^{2}$ NASA-Johnson Space Center \\ Mail Code SK \\ 2101 NASA Parkway \\ Houston, TX 77058
}

\begin{abstract}
This paper details a novel three-dimensional (3D) hydro-focusing micro cell sorter for micro flow cytometry applications. The unit was microfabricated by means of SU-8 3D lithography. The 3D microstructure for coaxial sheathing was designed, microfabricated, and tested. Three-dimensional hydrofocusing capability was demonstrated with an experiment to sort labeled tanned sheep erythrocytes (red blood cells). This polymer hydro-focusing microstructure is easily microfabraicted and integrated with other polymer microfluidic structures.
\end{abstract}

Keywords: SU-8, three-dimensional hydro-focusing, microfluidic, microchannel, cytometer

\section{Introduction}

Flow cytometric devices are very important for a wide range of biomedical research and clinical diagnostics. Conventional-sized flow cytometers are not novel and are widely used both in research and for clinical diagnostic purposes. Currently available commercial flow cytometers 
tend to be large and very expensive. The analytical sample is injected into the system, diluted, labeled, hydro-focused, and the cells are counted and sized by fluorimetric and electrical means. Figure 1 shows the principle of operation for the hydro-focusing unit of conventional flow cytomenters. The cells are labeled and driven to flow through a nozzle so that light scattering or fluorescence measurements can be used for analyses.

In recent years, one of the fast developing fields in science and engineering has been microelectromechanical systems (MEMS) technology. Many research efforts have been made in developing different types of micro-cytometry systems [1-12]. Micro-sized flow cytometry devices and components offer many potential benefits, including the ability to reduce device and sample sizes, development of low cost, single-use disposable devices, and improved device portability for field use along with low consumption of sample and buffer fluids, and reduction in the biohazard risk level.

In microfabricated flow cytometers, micro grooves are etched on a substrate such as silicon or glass. With a glass or polymer cover bonded on the top, micro channels are created to form a chamber with a size that permits cells to pass through a sensing unit for categorization and enumeration [1]. Because it is very difficult to develop a truly microfabricated cytometer, some researchers have tried to avoid the difficulties of complicated microfludic systems and micro optical systems. Weigl et al. [2-9] have used a simple design based on fluid/fluid extraction and developed a complete passive fluidic device that can be used to separate cells. The principle of hydro-focusing in a microchannel is based on the laminating cells with sheath flow. Their sample focusing system is only focused in the plane of substrate, not in the vertical direction between the top and bottom planes. In the vertical direction, fluid friction may make the cells not well focused. The cells along vertical direction therefore have different flow velocities.

G. Goranovic et al. [14] microfabricated a micro cell sorter with a "chimney" structure in silicon by reactive ion etching (RIE). Three-dimensional flow sheathing was obtained by injecting a sample into the sheath flow in a perpendicular direction. This design is difficult to integrate into other micro fluidic and micro optical measurement systems. Additionally, RIE microfabrication is an expensive approach. 


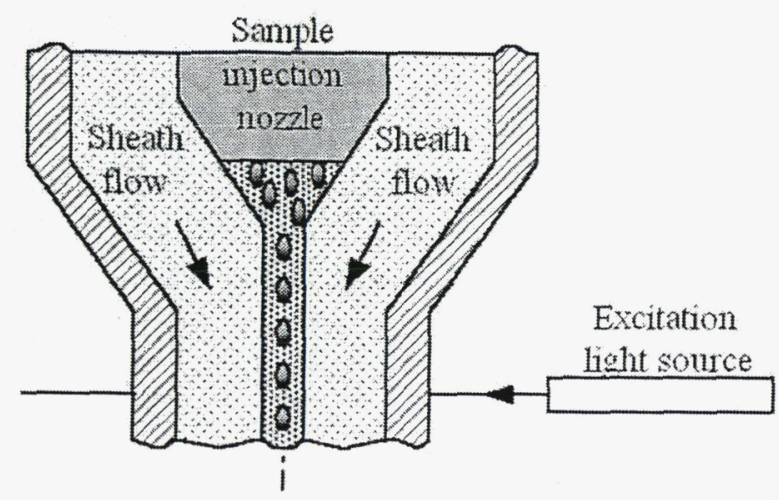

Figure 1. The operation principle of the conventional flow cytometer based on sample focusing and optical measurement principles

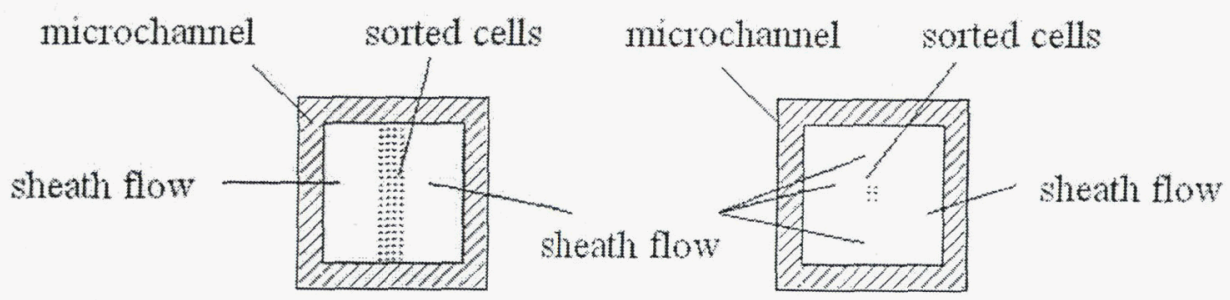
(A) 2D hydro-focus
(B) 3D hydro-focus

Figure 2. Difference in 2D versus 3D hydro-focusing in the cross-section of flow stream

For many biomedical/biochemistry applications, however, polymeric materials such as cured SU-8 offers unique advantages because of lower cost and good biocompatibility. Like most of the polymer-based systems, cured SU-8 may be used to construct complete systems rather than as simply a resist for the lithography process.

This paper presents a true three-dimensional hydro-focusing microstructure made within cured SU-8, which allows the cells to flow in the core stream with an almost uniform velocity.

\section{Design of the 3-D hydro-focusing Microfluidic Unit}

All conventional flow cytometers are designed to have a flow chamber with a nozzle, and are based on the principle of laminating cells with the sheath flow. The microfluidic unit in a flow cytometer needs to have cells in suspension flow within a single column (if possible) through an illuminated volume. In most instruments, this function is accomplished by injecting a 
small volume of sample flow through a small $(50-150 \mu \mathrm{m})$ nozzle into a much larger volume of sheath fluids. Both the sheath flow and sample flow require a small Reynolds number (when $\mathrm{R}_{\mathrm{e}}<2300$, flow is always laminar). A large volume of sheath flow compared with a small volume of sample flow will focus the sample flow along an axis. Figure 3 shows schematically how hydro-focusing occurs as a laminar flow enters a narrow channel. If the liquid is incompressible, nonviscous, and laminar flow, the flow passing section $A B$ will be focused on section $A^{\prime} B^{\prime}$. Under this lamination-flow and focusing condition, the sample in the core section will not mix with the sheath flow.

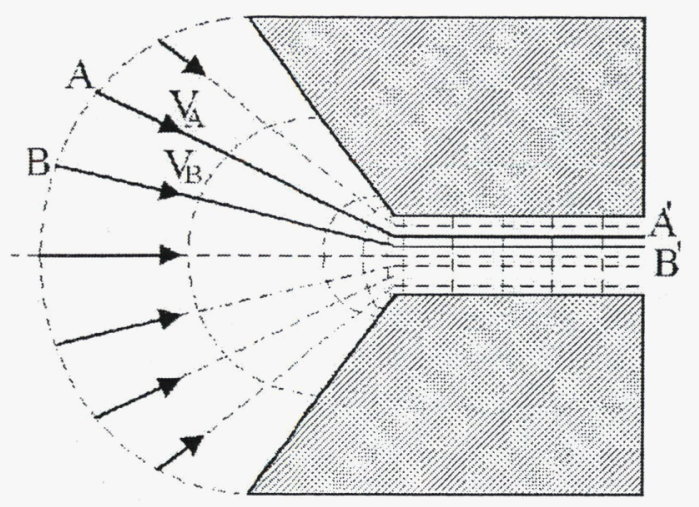

Figure 3. Laminar step flow

In order to achieve 3-dimensional hydro-focus, the microfluidic unit will inject the sample liquid and manipulate the flow rates to obtain a 3-dimensional pressure gradient. Sheath volume flow rate normally is much larger than the sample volume flow rate. In the micro flow cytometer, microchannels will determine the rate of sample and sheath flow, the focusing core stream, and the best focusing position. Also, the microchannel at the optimal focusing position will serve as the illumination point for fluorescent emission. Compared with the other three types of flow channel in flow cytometers (jet-in-air, closed-cross-flow, and open-flow-across-surface), microchannels with a flow-through cuvette have both cell a sorting function and excellent optical properties. Thus, it is the best choice for an integrated micro cytometer.

Based on the working requirements and the microfabrication limitations of UV lithography in SU-8, a 3D hydro-focusing unit for micro flow cytometry was designed as shown by the schematic diagram in Figure 3 (A). There are three inlets for the hydro-focusing microfluidic unit. Left-side and right-side inlets are for the sheath flow, the center inlet is for the 
sample flow. In the center of the end of this sample flow inlet, there is a diamond-shaped nozzle with a width of $100 \mu \mathrm{m}$. There are three slopes formed by tilt-exposed, cross-linked SU-8 polymer in which the center slope configured to a $30^{\circ}$ angle with the substrate. The flat covering glass and sloped-bottom help to focus the flow upward to a central region in the direction perpendicular to the substrate. The left-side slope, right-side slope, and the two sloped-sidewalls perpendicular to the substrate assist in achieving flow to the central region in a horizontal direction.

The sample flow is injected from the center inlet into the hydrofocusing chamber. Because of the symmetry of the left sloped-sidewall, the right sloped-sidewall, the left-side slope, and the right-side slope, the sample flow will be focused in the center axis of the outlet channel from the top view, as shown in Figure 4 (B). If the focusing chamber is sealed using a flat surface, the design is not symmetrical in the vertical direction, and thus the focused sample will be pushed upward, and there will be a slight off-set between the focused sample flow and the central axis of the outlet channel, as shown in Figure 4 (C). The outlet channel consists of cured SU-8 polymer and a glass plate, both of which have good optical properties and are suitable for an integrated micro optical measurement system to be incorporated in the future [15, 16].

Another option is to replace the flat covering plate with a modified structure similar to the bottom part. The only difference for the top part in comparison with the bottom one is that the sample injection nozzle is removed. This approach may slightly improve the focusing effect in the perpendicular direction. However, it also increases the difficulty of assembly.

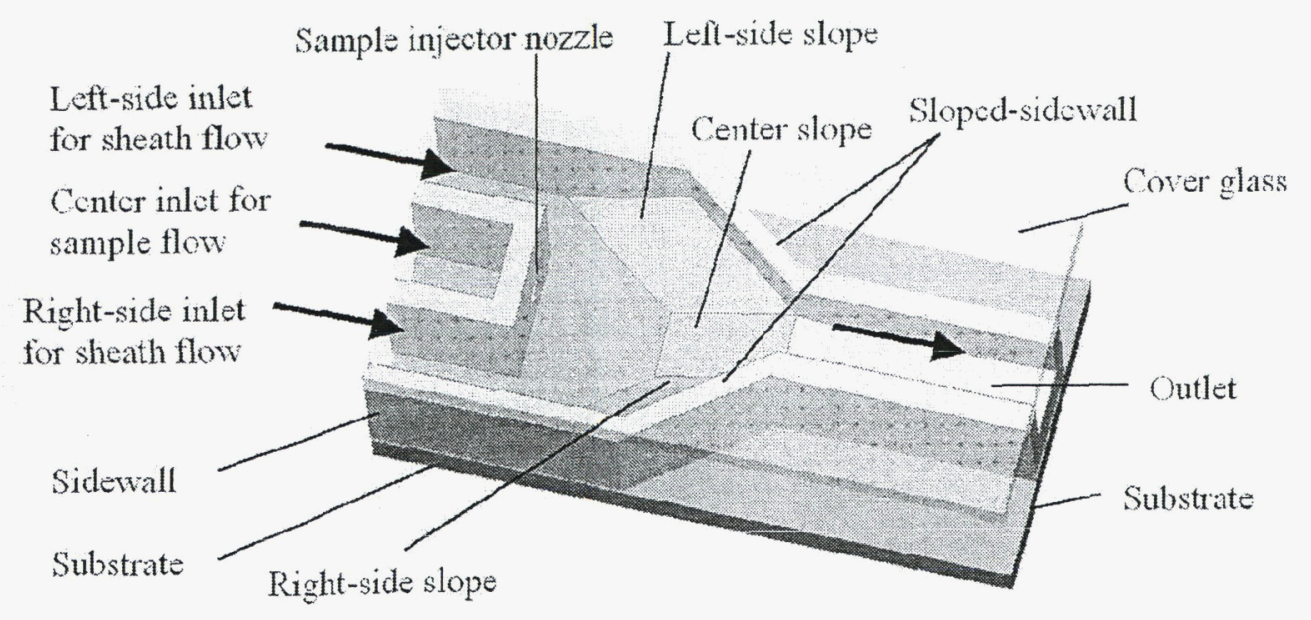

(A) 


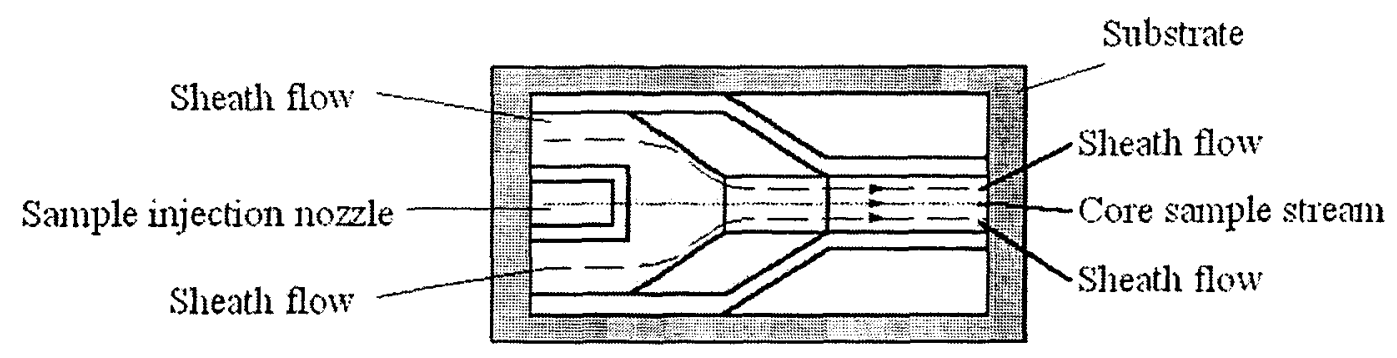

(B)

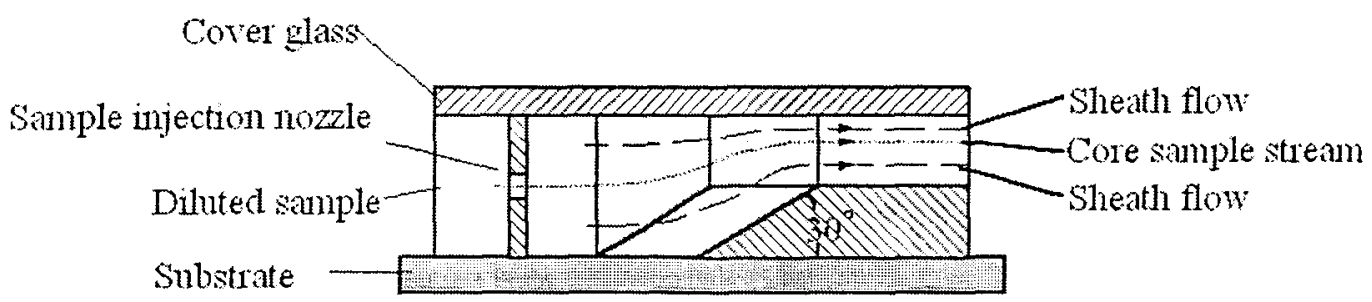

(C)

Figure 4. (A) Schematic of the 3D hydro-focusing unit (B) Top view of the hydrofocusing

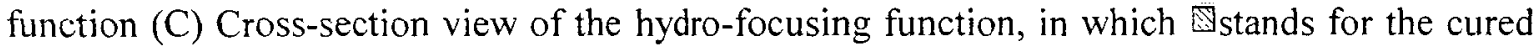
SU-8, 7 stands for the substrate, 图 stands for the cover glass.

\section{Microfabrication of the 3-D hydro-focusing unit}

The 3-D hydro-focusing unit was fabricated using UV lithography of SU-8. Cured SU-8 is a physically strong polymer and is well suited as a structural material for this application. It has excellent thermal stability and is highly resistive to a wide variety of chemicals. It is also biocompatible and can be treated with other types of biomaterials such as parylene, if desired. The process used in fabrication of this $3 \mathrm{D}$ hydro-focusing unit is also compatible with other micromachining processes and can be easily integrated with other processes to create complicated micro fluidic systems.

A total of three lithography masks were needed. The first was used to form the three slope surfaces; the second was used to make the sample injector nozzle; and the last one was used to fabricate the channel sidewalls.

The sloped surfaces of SU-8 polymer in the hydro-focusing unit were fabricated using a non-conventional lithography method in which a thick layer of SU-8 was exposed at a specific 
tilted angle with respective to the substrate surface. Because SU-8 is a negative resist, exposed regions were kept and unexposed regions were removed in the development process.

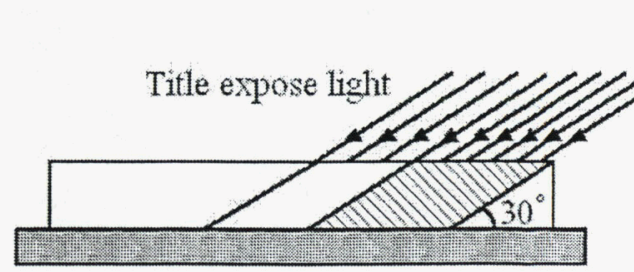

Cross section view

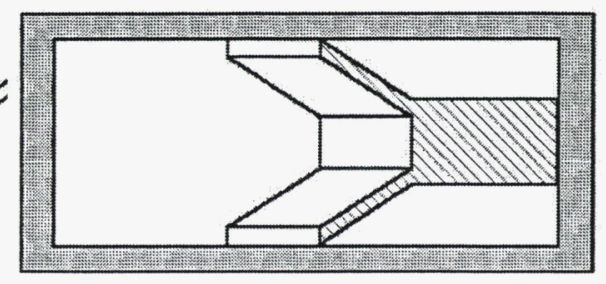

Top view

(A)

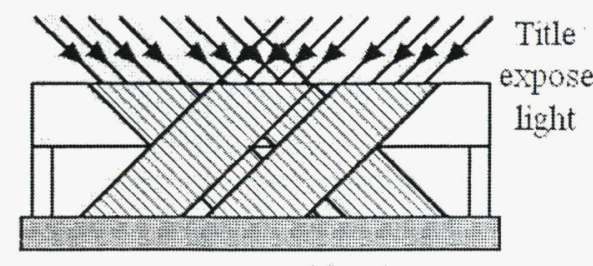

Side view

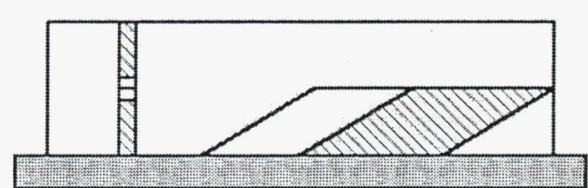

Cross section view

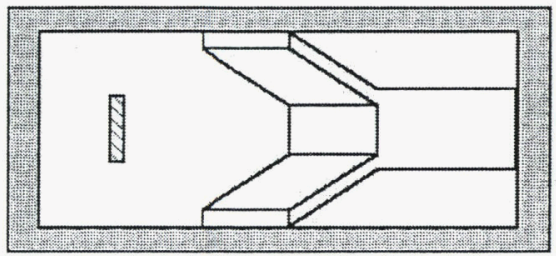

Top view

(B)

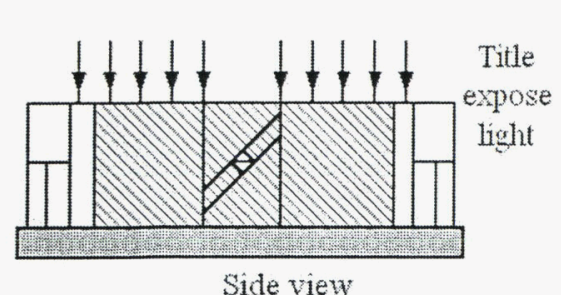

Side view

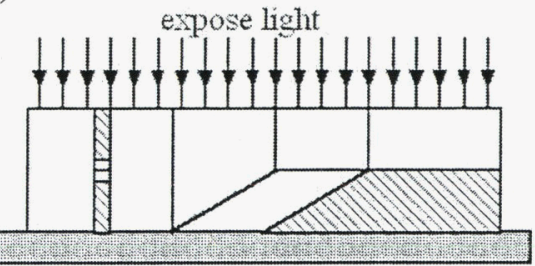

Cross section view

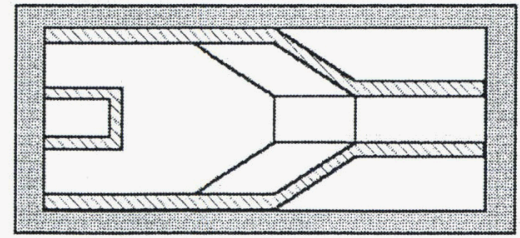

Top view

(C)

Figure 5. (A) $60^{\circ}$ angle tilt exposes SU-8 to obtain slopes having $30^{\circ}$ angle with the substrate (B) $45^{\circ}$ angle tilt exposes SU-8 to obtain suspended sample injector nozzle in the center position of 
the sample inlet end (C) expose all of SU-8 sidewalls. In (A), (B) and (C), 罗stands for the cured

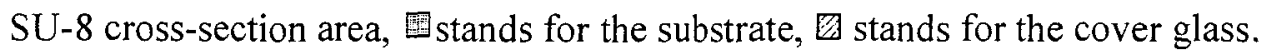

The fabrication process for the hydrofocusing unit is schematically shown in Figure 5 (A) and (B). A specially designed chuck was used to hold the mask and the substrate. The chuck permits a rotation angle of up to 90 degrees. As shown graphically in Figure $5(\mathrm{~A})$, when a tilted light beam is projected on the photoresist at 60 degrees respective to the substrate, three sloped surfaces are formed. In the second step of the fabrication as shown in Figure 5 (B), the resist was exposed at 45 degree and -45 degree respectively with the designed mask, with two narrow strips of light beams projected on the photoresist. After development, the intersection forms a diamond-shaped sample injection nozzle (center inlet in Figure 4(A)). Finally, conventional lithography was utilized to fabricate the inlet and outlet flow channels in Figure $5(\mathrm{C})$. This last step aids in fabricating all of the vertical sidewalls as shown in the diagrams of Figure 4.

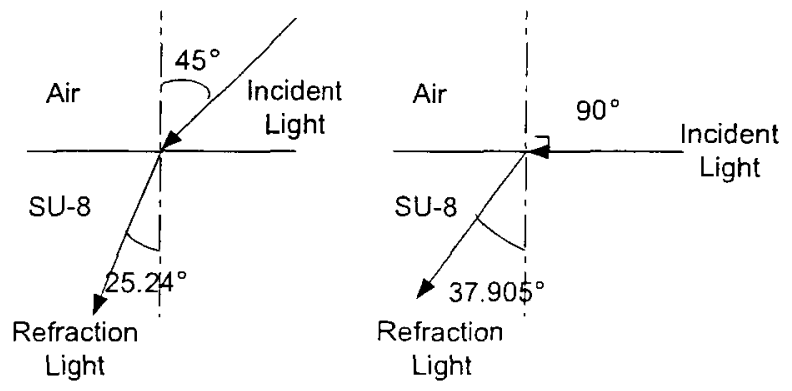

Figure 6. The refraction of the SU-8 resist reduced angle of the light projection. SU-8's refraction and the critical angle (critical angle is about $37.9^{\circ}$ at $365 \mathrm{~nm}$ )

The basic principle of tilted lithography for SU-8 can be explained using the schematic diagram in Figure 6. Because the refraction of the light at the surface of the SU-8 resist, a light beam projected on the resist at 45 degrees may propagate at a reduced angle. Based on the refraction index of SU-8 $(\mathrm{n}=1.668$ at $\lambda=365 \mathrm{~nm}, \mathrm{n}=1.650$ at $\lambda=405 \mathrm{~nm})$, it was calculated to be roughly at about 25.08 degrees as shown in Figure 6. In some applications, if the channel's designs needs to obtain the intersections with degrees greater than 37.905 degrees, a coupling prism and optical liquid are required to compensate for this refraction [15][16][17] to obtain a square-shaped injector nozzle and slopes. 
If the diamond-shaped nozzle with an intersection less than 37.905 degrees is needed, the lithography can be done without compensation provided by a coupling prism and optical liquid.

If a 90 degrees intersection is required to obtain a square-shaped sample injector nozzle, the required prism and the compensation principle [15][16] are shown in Figure $7(\mathrm{~A})$. From the diagram, for $\lambda=365 \mathrm{~nm}$ the following calculation can be made:

If $\theta_{1}$, the anglc at which the light enters SU- 8 resist, needs to be $45^{\circ}$, from Snell's law,

$$
n_{1} \sin \theta_{1}=n_{2} \sin \theta_{2}=n_{3} \sin \theta_{3}=n_{4} \sin \theta_{4}=n_{5} \sin \theta_{5},
$$

$\theta_{5}$ can be obtained as

$$
\theta_{5}=\sin ^{-1}\left(\frac{n_{1} \sin \theta_{1}}{n_{5}}\right)=\sin ^{-1}\left(\frac{1.668 \cdot \sin 45^{\circ}}{1.53}\right)=50.43^{\circ} .
$$

From the geometric relationship, we know

$$
\theta_{6}=\theta_{5}-45^{\circ}=50.43-45=5.43^{\circ} \text {. }
$$

From Snell's law, $\theta_{7}$ the following can be obtained:

$$
\theta_{7}=\sin ^{-1}\left(\frac{n_{6} \sin \theta_{6}}{n_{7}}\right)=8.32^{\circ}
$$

The substrate therefore needs to be kept $45+8.32=53.32^{\circ}$ with the horizontal level and with the positional relationship as shown in Figure 7 (A) to completely compensate for the refraction at the interface to obtain a 90 degrees intersection as required for a square-shaped injector nozzle.

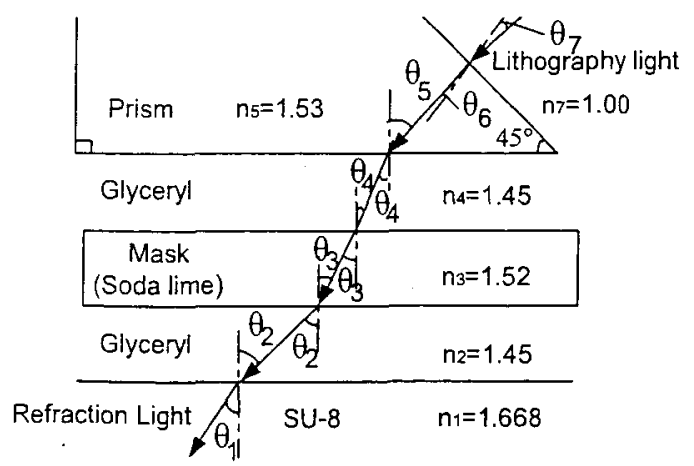

(A)

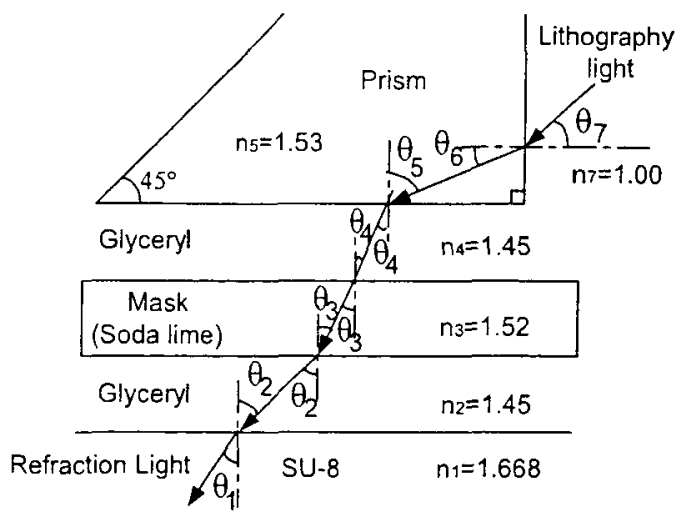

(B)

Figure 7. Positions of the prism, mask, SU-8 layer and the substrate 
To obtain the 30 degrees slopes with the substrate, if the position relationship is still kept as in Figure $7(\mathrm{~A})$, with the equations (1) to (6), $\theta_{7}$ is calculated as $41.67^{\circ}$. Thus, the substrate needs to be maintained at $86.674^{\circ}$ with the horizontal level. This angle is too big to work in the processing conditions. If the position changes to Figure 7 (B), the substrate's tilt angle will be reduced to a reasonable range under the processing condition.

If $\theta_{1}$, the angle at which the light enters SU-8 resist, needs to be $60^{\circ}$, from Snell's law as equation (3), $\theta_{5}$ can be obtained as

$$
\theta_{5}=\sin ^{-1}\left(\frac{n_{1} \sin \theta_{1}}{n_{5}}\right)=\sin ^{-1}\left(\frac{1.668 \cdot \sin 60^{\circ}}{1.53}\right)=70.76^{\circ}
$$

From geometry relationship, we know

$$
\theta_{6}=90^{\circ}-\theta_{5}=90^{\circ}-70.76^{\circ}=19.24^{\circ} \text {. }
$$

From Snell's law, $\theta_{7}$ can be obtained:

$$
\theta_{7}=\sin ^{-1}\left(\frac{n_{6} \sin \theta_{6}}{n_{7}}\right)=30.279^{\circ}
$$

The substrate therefore needs to be kept $90-30.28=59.72^{\circ}$ with respective to the substrate and with the position relationship as shown in Figure 7 (B) to completely compensate for the refraction at the interface to obtain a $60^{\circ}$ incident angle in SU-8 (to get $30^{\circ}$ slopes with the substrate).

The fabrication step is as follows: (1) clean the Si wafer or glass substrate; (2) spin-coat SU-8 100 photo resist with $890 \mathrm{rpm}$ to obtain 500 om thick resist layer; (3) pre-bake at $96^{\circ} \mathrm{C}$ for 5 hours, cool down to $60^{\circ} \mathrm{C}$ within 1 hour and remain at $60^{\circ} \mathrm{C}$ for 3 hours, then cool down to room temperature in 2 hours; (4) conduct a $60^{\circ}$ tilted-exposure of SU-8 with the help of prism and optical liquid for refraction compensation. This produced slopes tilted $30^{\circ}$ angle with the substrate $[15,16]$ as shown in Figure $5(\mathrm{~A}) ;(5)$ post-bake the sample at $96^{\circ} \mathrm{C}$ for 20 minutes, cool down to $60^{\circ} \mathrm{C}$ in 1 hour and remain at $60^{\circ} \mathrm{C}$ in 3 hours, then cool down to room temperature in 2 hours; (6) spin coat SU-8 100 photo resist with $890 \mathrm{rpm}$ to obtain the second $500 \mathrm{~cm}$ thick resist layer; (7) pre-bake the sample at $110^{\circ} \mathrm{C}$ for 5 hours, cool down to $60^{\circ} \mathrm{C}$ in 1 hour and maintain at $60^{\circ} \mathrm{C}$ for 5 hours, then cool down to room temperature in 3 hours; (8) $45^{\circ}$ angle 

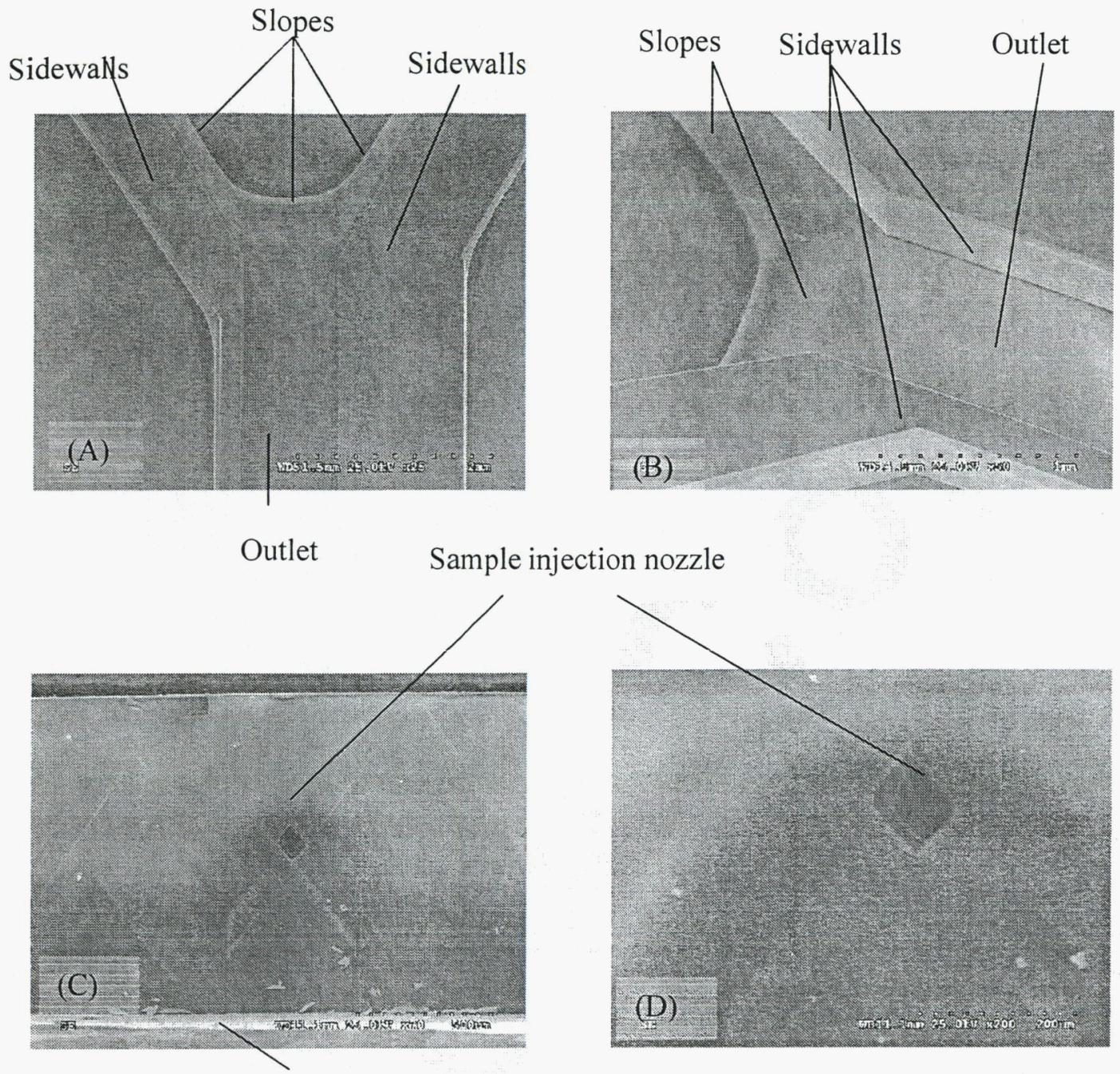

Substrate

Figure 8. SEM photographs of the fabricated hydrodynamic focusing unit

tilted-exposure of SU-8 with correction prism and optical liquid to obtain suspended sample injector nozzle in the center of the sample inlet end $[15,16]$, as shown in Figure 5 (B); (9) expose all of the SU-8 sidewalls, as shown in Figure $5(\mathrm{C})$; $(10)$ post-bake samples at $110^{\circ} \mathrm{C}$ for 20 minutes, then cool down to $60^{\circ} \mathrm{C}$ in 1 hour and remain at $60^{\circ} \mathrm{C}$ for 5 hours, then cool down to room temperature in 3 hours; (11) development in SU-8 developer for 2 hours, rinse with IPA and DI water, dry in air; (12) bond cover glass, seal inlet and outlet tubes. The microfabricated hydrofocusing unit (without covering glass) is as shown in Figure 8. 
The total dimensions of this hydrofocusing unit is about $10 \mathrm{~mm}$ long, and $8 \mathrm{~mm}$ wide. The inlet for sheath flow and center inlet for sample flow of the fabricated hydrofocusing unit are $1 \mathrm{~mm}$ wide. On a single wafer, sample injection nozzles with several different sizes have been tried, $100 \mu \mathrm{m}, 200 \mu \mathrm{m}$, and $150 \mu \mathrm{m}$. The outlet channel is $500 \mu \mathrm{m}$ deep. The outlet channel's width is in a series with $100 \mu \mathrm{m}, 300 \mu \mathrm{m}, 500 \mu \mathrm{m}$ and $1000 \mu \mathrm{m}$.

The outlet channel of the prototype unit as presented in this paper is $1 \mathrm{~mm}$ wide and $500 \mu \mathrm{m}$ deep; the diagonal width of the sample injection nozzle is $150 \mu \mathrm{m}$.

\section{Experiments and Discussions}

The experimental setup is schematically shown in Figure 9. Three BD plastic syringes were seated on a syringe pump. For testing purpose, the sample flow was injected using a $1 \mathrm{cc}$ BD plastic syringes. Sheath flow was pumped in with two 20cc BD plastic syringes. Two tests were conducted. In the first experiment, a fluorescent dye (Aldrich Chemical Company, Inc. Catalog \# F245-6) solution with $1.2 \mathrm{mMol} / \mathrm{L}$ concentration was used as the sample flow and DI water was used as the sheath flow. This test was used to check the hydrofocusing capability of the prototype. The second experiment was conducted to check the functionality of the prototype in sorting microscopic objects such as red blood cells. In the second test, diluted, labeled, tanned sheep erythrocytes (red blood cells) were used as the sample and a saline solution was used as the sheath flow.

The three syringes used were of the same length, but had different cross-sectional areas. The 20cc BD syringes' cross-section is 20 times that of the $1 \mathrm{cc}$ syringe. A syringe pump (Harvard Apparatus' PicoPlus) was used to drive all three syringes at the same speed. The flow rates of the left-side sheath flow, the right-side sheath flow, and the sample flow at the center are therefore in the ratios of $20: 20: 1$. A change of syringe diameters (syringe volume) would change the sheath-sample ratio. Sample solution and sheath flow were pumped through the inlets into the hydrofocusing unit. The three-dimensional laminar sheath flow hydro-focused the sample flow into the core stream of the outlet micro-channel. A mercury lamp was used to provide illumination through the microscope onto the outlet microchannel. The emission light and the reflected light from the outlet microchannel were filtered using an optical filter, which permitted mainly the emission light to pass through. The images of focusing flow were magnified with a microscope. A Nikon CV-252 digital video camera was used to monitor the hydrofocusing 
process. This standard NTSC video camera collects 30 frames (actually 29.97) per second, and two fields per frame.

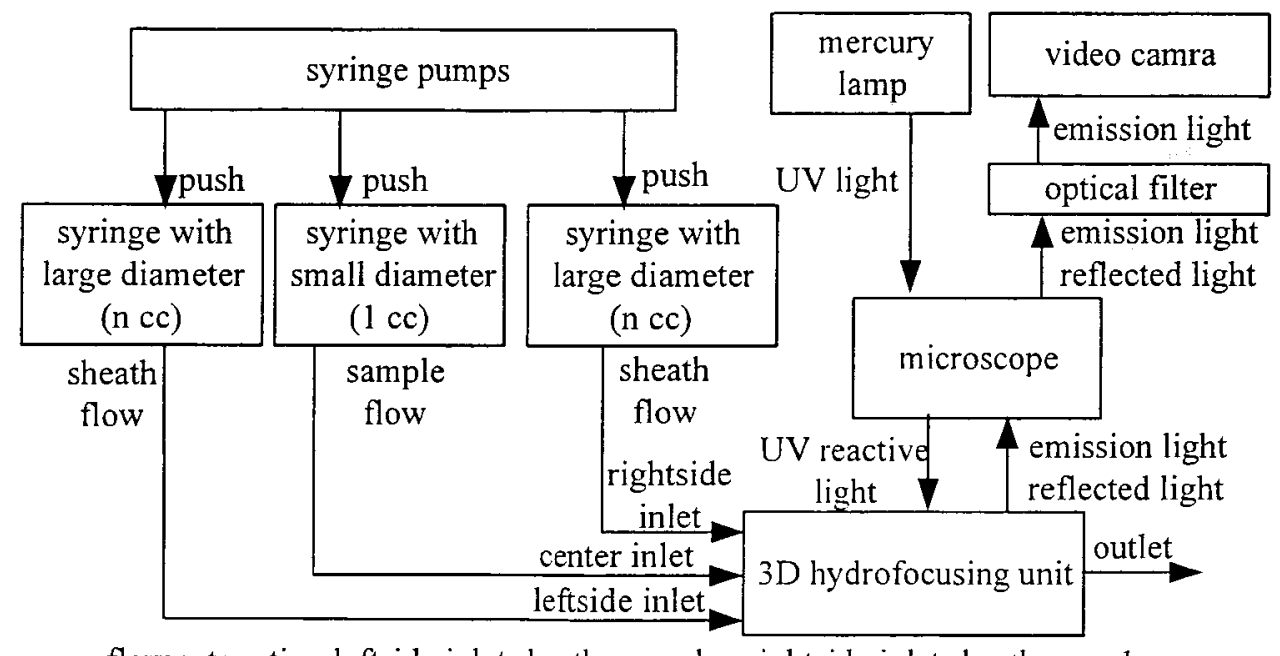

flow rate ratio: leftside inlet sheath: sample: rightside inlet sheath $=n: 1: n$

Figure 9. Experiment setup

In the first experiment (using a fluorescent dye), the dye solution was pumped from the center inlet through the injector nozzle into the hydro-focusing chamber, DI water (sheath flow) was pumped from the right and left inlets into the hydro-focusing chamber. The threedimensional hydro-focusing effect can be measured by inspection of the gray scale distribution in the photo images of the CCD video camera. Single frames were selected from the hydrofocusing video by Adobe Premiere Pro software [Adobe Systems Incorporated, San Jose, $\mathrm{CA}]$. In the pictures of the hydro-focusing chamber, the region with high concentration of fluorescent dye (sample flow) are brighter than that without fluorescent dye and thus (sheath flow) would be dark. The hydro-focusing function in the horizontal direction was shown in Figure 10. The video camera and microscope used in the experiments has very limited depth of focus (DOF). The bright images of the center core stream would disappear when the video camera and microscope were adjusted up or down away from the position to take the pictures as in Figure 10. This means the flow with high concentration of fluoresent dye was only in one level and it was focused in the vertical direction.

In the second test, diluted solution of labeled tanned sheep erythrocytes (red blood cells) was used as sample flow and the saline water was used as the sheath flow. A single frame picture was selected from the video of hydrofocusing labeled erythrocytes by Adobe Premiere Pro 
software as shown in Figure 11. It shows an image of the labeled tanned sheep erythrocytes (red blood cells) as they were focused and sorted in the chamber of the three-dimensional hydrofocusing unit. A black dot was marked on the cover glass to help to identify the central region of the flow chamber. From the photograph in Figure 11, it can be seen that cells were lined up along the central line in the photograph. Because this standard NTSC video camera is only capable of catching 30 frames of images (actually 29.97) per second, the image quality dramatically reduced when a single frame is taken out from the movie video. The movement of these cells were clearly visible in the video. However, only faint images of these high-speed moving cells could be seen in the picture. The edges of cell images in the picture are not very clear, the images of the labeled erythrocytes are circled in the figure to help identify their positions.

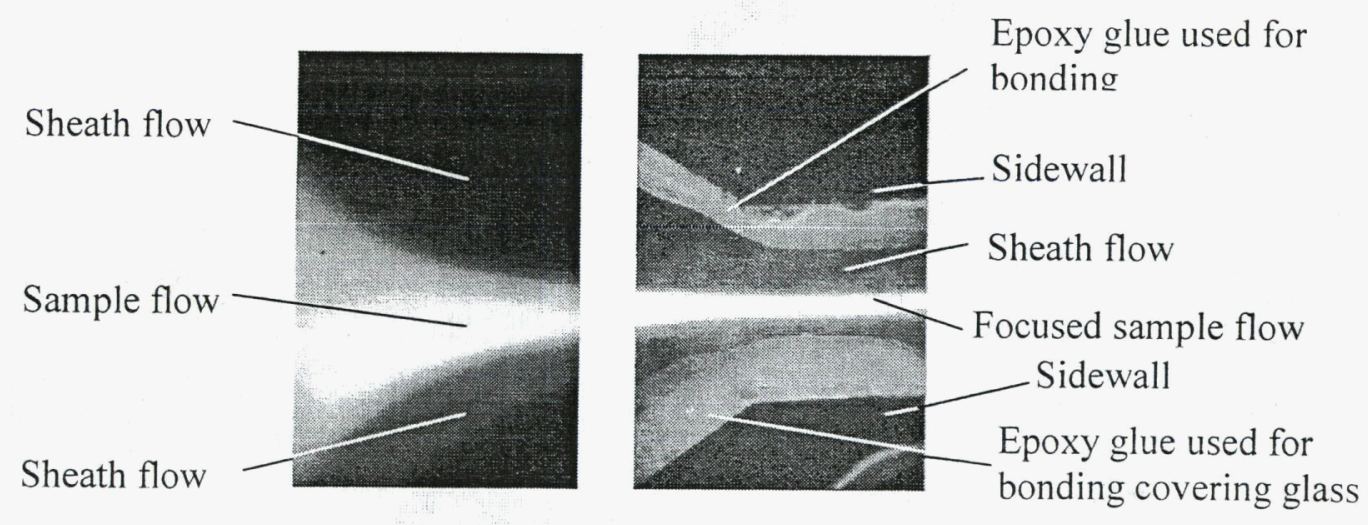

Figure 10, Images of fluoresce dye solution was focused (x4)

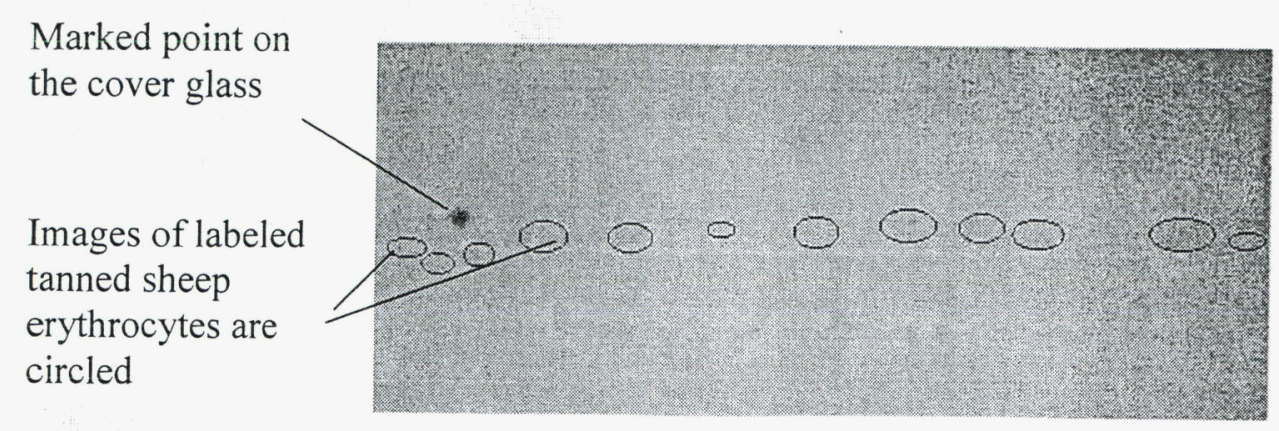

Figure 11, Cell sorting (x10) images of hydro-focusing unit. The image quality dropped dramatically when a single frame is taken out from the video taken with NTSC CCD camera. 
The erythrocytes (red blood cells) used in the test are microscopic biconcave disks with a diameter approximately 6-10 $\mathrm{cm}$. When these cells were hydro-dynamically focused in the chamber of the prototype unit, any slight differences in flow velocity across the stream may pull the cells to rotate around and be aligned with the direction of the stream. Eventually, all the cells were pulled to orient their long axis along the stream to achieve dynamic balance. The shear stresses in the flow also cause cells to be elongated along their long axes. Based on video of hydrofocusing labeled erythrocytes, all sample cells were animatedly oriented, deformed, and focused by the hydrodynamics force.

\section{Conclusions}

A prototype of a truly three-dimensional hydrodynamic focusing unit for use in an integrated microcytometer was successfully microfabricated. It proved that it is feasible to utilize 3-D lithography technology to fabricate the device with cured SU-8 polymer as the structural material. The experiments with labeled tanned sheep erythrocytes (red blood cells) have proved that sample flow was very effectively focused and cells were neatly lined up along the axial line of the central stream. This unit can be integrated with other micro flow and micro optical systems to be incorporated into a micro-sized blood cell categorizing and counting system for use during space flight and in other remote point-of-care applications including military field operations. The simple fabrication technology is based on 3-D lithography of SU-8 and uses cured SU-8 as the structural material. This simple approach results in a very low fabrication cost. Further study needs to be conducted in the near future to better understand the fluid dynamics in the micro-chamber for optimal design.

\section{Acknowledgments}

The authors would like to thank Dr. Soper and his group in Chemistry Department of Louisiana State University for helping to setup the instruments for the experiments about threedimensional hydrofocus tests. 


\section{Reference}

1. Anne Y. Fu, Charles Spence1, Axel Scherer, Frances H. Arnold, and Stephen R. Quake,"A microfabricated fluorescence-activated cell sorter," Nature Biotechnology Vol. 17 November 1999, pp. 1109-1111.

2. B. H. Weigl, C. J. Morris, N. Kesler, C. F. Battrell, R. L. Bardell, "Standard and highthroughput microfluidic disposables based on laminar fluid diffusion interfaces," SPIE BIOS 2002, San Jose, January 2002.

3. B. H. Weigl and K. Hedine, "Lab-on-a-chip-based separation and detection technology for life science applications," American Biotechnology Laboratory, Jan. 2002, Vol. 20:1 pp. 2830 .

4. B.H. Weigl, R. L. Bardell, T. H. Schulte, C. F. Battrell, J. Hayenga, "Design and Rapid Prototyping of Thin-Film Laminate-Based Microfluidic Devices," Biomedical Microdevices 2001, Vol. 3:4 pp. 267-274, 2001, Kluwer Academic Publishers.

5. B. H.Weigl "Microfluidics-based lab-on-a-chip systems", IVD Technology Nov/Dec 2000, vol. 6: pp.47-53, 2000.

6. B. H. Weigl, A. Hatch, A. Kamholz, P. Yager, "Novel Immunoassay Formats for Integrated Microfluidic Circuits - Diffusion Immunoassays (DIA)," Micro-\& Nanotechnology for Biomedical and Environmental Applications, SPIE 2000, Proceedings of SPIE, 3912, pp.5056.

7. B. H. Weigl, R. L. Bardell, T. H. Schulte, C. Williams, "Passive Microfluidics- UltraLow-Cost Plastic Disposable Lab-on-a-chips," $\mu$ TAS 2000 Proceedings, pp.299, 2000, D. J. Harrison and A. van den Berg, eds. Kluwer Academic Publishers, Dordrecht, (2000).

8. Ron L Bardell, Bernhard H Weigl, Natasa Kesler, Thomas Schulte, Jon Hayenga, Fred Battrell, "Microfluidic Disposables for Cellular and Chemical Detection - CFD Model Results and Fluidic Verification Experiments," SPIE BIOS 2001 - Proc. SPIE 4265-02, 2001.

9. Ron L Bardell, Bernhard H., Natasa K., Thomas S., Jon H. and Fred B., "Microfluidic Disposables for Cellular and Chemical Detection - CFD Model Results and Fluidic Verification Experiments," SPIE BIOS 2001 - Proc. SPIE 4265-02, 2001. 
10. Gwo-Bin Lee, Che-Hsin Lin, Guan-Liang Chang, "Micro flow cytometers with buried SU-8/SOG optical waveguides," Sensors and Actuators A 3559 (2002) pp.1-6.

11. J. Yang et al, "Cell Separation on Microfabricated Electrodes Using Dielectrophoretic/ Gravitational Field-Flow Fractionation," Anal. Chem. 71, pp. 911-918.

12. G. Medoro et al., "CMOS-only sensor andmanipulator for microorganisms," Proceedings of International Electron Dcvicc Mccting (IEDM), pp.415-418, 2000.

13. Haibo Li and Rashid Bashir, "Dielectrophoretic separation and manipulation of live and heat treated cells of Listeria on microfabricated devices with integrated electrodes," Sensors and Actuators B 86 (2002) pp.215-221.

14. G. Goranovic, I. R. Perch-Nielsen, U.D. Larsen, A. Wolff, J. P. Kutter, P. Telleman, "Three-Dimensional Single Step Flow Sheathing in Micro Cell Sorters", Modeling and Simulation of Microsystems 2001, (www.cr.org), ISBN 0-9708275-0-4, P242-245

15. Ren Yang, Wanjun Wang, "Out-of-plane polymer refractive microlens fabricated based direct lithography of SU-8", Sensors and Actuators A: Physical, article in press.

16. Ren Yang, John Williams, Wanjun Wang, "A Rapid Micro-mixer/reactor Based on Arrays of Spatially Impinging Micro-jets" Journal of Micromechanics and Microsystems, article in press.

17. Ren Yang, Wanjun Wang, "Numerical and Experimental Study on an Out-of-Plane Prealigned Refractive Microlens Fabricated Using UV Lithography Method", Optical Engineering, article in press 